\title{
Ectopic expression of Smurf2 and acceleration of age-related intervertebral disc degeneration in a mouse model
}

\author{
Qiuqian Wu, PhD, and Jason H. Huang, MD \\ Department of Neurosurgery, Institute for Translational Medicine, Baylor Scott \& White Health System, Temple, Texas
}

OBJECTIVE Lumbar intervertebral disc degeneration, an age-related process, is a major cause of low-back pain. Although low-back pain is a very common clinical problem in the aging population, no effective treatment is available, largely owing to lack of understanding of the molecular mechanisms underlying disc degeneration. The goal of this study was to characterize how ectopic expression of Smurf2 driven by the collagen Type II alpha 1 (Col2a1) promoter alters disc cell phenotype and associated cellular events, matrix synthesis, and gene expression during disc degeneration in mice.

METHODS To characterize how ectopic expression of Smurf2 in Col2a1-promoter working cells affects the disc degeneration process, the authors performed histological and immunohistochemical analysis of lumbar spine specimens harvested from wild-type (WT) and Col2a1-Smurf2 transgenic mice at various ages ( $\mathrm{n} \geq 6$ in each age group). To elucidate the molecular mechanism underlying Smurf2-mediated disc degeneration, the authors isolated cells from WT and Col2a1-Smurf2 transgenic lumbar intervertebral discs and performed Western blot and real-time RT-PCR (reverse transcription polymerase chain reaction) to examine the protein and mRNA levels of interesting targets.

RESULTS The authors demonstrated that approximately $30 \%$ of WT mice at 10-12 months of age had started to show disc degeneration and that the disc degeneration process was accelerated by 3-6 months in Col2a1-Smurf2 transgenic mice. Chondrocyte-like cell proliferation, maturation, and fibrotic tissue formation in the inner annulus were often accompanied by fibroblast-to-chondrocyte differentiation in the outer annulus in transgenic discs. The chondrocyte-like cells in transgenic discs expressed higher levels of connective tissue growth factor (CTGF) than were expressed in WT counterparts.

CONCLUSIONS The findings that ectopic expression of Smurf2 driven by the Col2a1 promoter accelerated disc degeneration in Col2a1-Smurf2 transgenic mice, and that higher levels of CTGF protein and mRNA were present in Col2a1Smurf2 transgenic discs, indicate that Smurf2 accelerates disc degeneration via upregulation of CTGF.

https://thejns.org/doi/abs/10.3171/2016.11.SPINE16901

KEY WORDS Smurf2; Col2a1 promoter; intervertebral disc degeneration; connective tissue growth factor; CTGF

$\mathrm{C}$ ARTILAGE is a specialized type of connective tissue. Depending on its location in vivo, cartilage functions as growth plate cartilage, fibrocartilage, or articular cartilage. Growth plate cartilage is transient and is responsible for long-bone lengthening during development via the process of endochondral ossification, which consists of mesenchymal cell-chondrocyte differentiation (chondrogenesis), chondrocyte proliferation and matrix synthesis, chondrocyte maturation into hypertrophic chondrocytes, and replacement of hypertrophic tissue by bone. Articular cartilage covers the ends of long bone and functions in a stable state throughout postnatal life. ${ }^{5,11,29,}$ ${ }^{30,44}$ However, during osteoarthritis, articular chondrocytes recapitulate the maturation process that normally occurs in the growth plate during endochondral ossification,,$^{16,40,45}$ leading to articular cartilage degradation. Fibrocartilage is uniquely situated within specific joint spaces to ensure smooth articulation and stress absorption. For example, menisci in knee joints and discs in intervertebral joints are the major fibrocartilage tissues found in the human body.

ABBREVIATIONS Col2a1 = collagen Type II alpha 1; CTGF = connective tissue growth factor; $\mathrm{OCT}=$ optimal cutting temperature compound; RT-PCR = reverse transcription polymerase chain reaction; TGF $\beta=$ transforming growth factor $-\beta$; WT = wild type.

SUBMITTED July 29, 2016. ACCEPTED November 14, 2016.

INCLUDE WHEN CITING Published online April 7, 2017; DOI: 10.3171/2016.11.SPINE16901. 
They possess complex geometrical shapes with highly organized matrix structures, which are necessary for proper tissue function.

The meniscus exhibits regional and zonal variations in its cellular composition and microstructure. ${ }^{15,18,35}$ The meniscus is wedge shaped and located at the periphery of the articular surface of the tibia. ${ }^{24}$ The outer two-thirds of the meniscus is fibrocartilage, where fibroblastic cells are fusiform in shape and mainly express Type I collagen and small amounts of Type II collagen and proteoglycans. The inner one-third of the meniscus consists of articular cartilage tissue in which the rounded chondrocytes balance the slow matrix turnover. ${ }^{36,39}$

Similar to menisci, intervertebral discs also vary in cell phenotype and matrix structure from one region to another., ${ }^{9,10,31,34}$ An intervertebral disc consists of an annulus fibrosus ring, a nucleus pulposus core, and 2 endplates. The outer annulus is made up of highly ordered collagen lamellae in which Type I collagen fibers are aligned with elongated fibroblastic cells. ${ }^{34}$ The inner annulus differs from the outer section, containing spherical cells, more widely spaced layers, and greater amounts of Type II collagen and proteoglycans.9,31 The central nucleus, a hydrogel-like tissue, is predominantly composed of proteoglycans and Type II collagen. The highly negatively charged glycosaminoglycan in the nucleus provides osmotic properties that enable the nucleus to maintain height and turgor against compressive loads..$^{23,42}$ The nucleus cells that produce and maintain the nucleus matrix are larger clusters of notochordal cells and relatively smaller chondrocyte-like cells. ${ }^{23}$ The endplate, situated at the articular surface of the intervertebral disc and the adjacent vertebrae, ${ }^{20}$ is a layer of articular cartilage-like tissue containing chondrocytelike cells embedded within a cartilage matrix. The annulus, the nucleus, and the endplates are interconnected to form the most important part of the motion segment of the spine.

Low-back pain, a very common clinical problem, is frequently caused by lumbar intervertebral disc degeneration. ${ }^{12,14,20}$ Disc degeneration begins as early as the 2 nd decade of life and is an inevitable consequence of aging. ${ }^{4}$ Thus, it is difficult to distinguish the physiological process of disc aging from that of degeneration; however, when structural failure of a disc is observed in combination with accelerated or advanced signs of aging, the disc is considered to be a degenerative disc. ${ }^{2}$ The process of disc aging/ degeneration has been clearly described at macroscopic and histological levels. For example, with increasing age, water content decreases and collagen content increases in the nucleus. Clefts and tears first occur in the nucleus and endplates and then extend to the annulus, and loss of the annulus-nucleus boundary and the superficial layer of the endplates, fibroblast-chondrocyte differentiation, chondrocyte cloning, and annulus migration into the nucleus are frequently detected in aging/degenerating discs. In the late stage of disc degeneration, unsuccessful repair of structural failure leads to the formation of scar or fibrotic tissue, a process that results in the progression of structural failure rather than repair., ${ }^{4,27,33}$ Little is known about the regulation of disc aging or the progression of structural failure. It is known that connective tissue growth factor
(CTGF) and its expression inducer transforming growth factor $-\beta$ (TGF $\beta$ ) are the key factors for regeneration of connective tissue during cutaneous wound healing and fibrosis formation when high CTGF expression by fibroblasts persists. ${ }^{17,19}$ However, it is not clear whether CTGF expression is altered in degenerative disc cells or in the cells that are prone to become degenerative or fibrotic tissue during disc aging or degeneration.

We have previously shown that ectopic expression of Smurf2, an E3 ubiquitin ligase, under the control of collagen Type II alpha 1 (Col2al) promoter induces osteoarthritis in adult knee joints of Col2al-Smurf2 transgenic mice ${ }^{45}$ We found that when articular cartilage underwent chronic degeneration in Col2al-Smurf2 transgenic mice, similar alterations occurred simultaneously in meniscus. Thus, we reasoned that Col2al-Smurf2 transgenic mice might exhibit a disc degeneration phenotype. The goal of this study was to characterize how ectopic expression of Smurf2 in Col2al-promoter working cells in mice altered disc phenotype and associated mechanisms.

\section{Methods \\ Col2a1-Smurf2 Transgenic Mice}

We previously generated 3 lines of Col2al-Smurf 2 transgenic C57BL/6-SJL mice by cloning FLAG-tagged human Smurf2 cDNA downstream collagen Type II alpha 1 (Col2al) promoter. ${ }^{45}$ We recently used materials and methods similar to those used in our previous study ${ }^{45}$ including FLAG-tagged Smurf2 cDNA (Addgene) and Col2al promoter (Yoshihiko Yamada, NIH/NIDCR), except that the Smurf2 cDNA fragments were injected into fertilized C57BL/6 oocytes (Cyagen Biosciences). The cells with ectopic Smurf2 expression driven by the Col2al promoter in Col2al-Smurf2 transgenic mice were identified by immunostaining with anti-FLAG M2 antibodies (Sigma). The positive cells for this staining were chondrocytes, osteoprecursors, and fibrocartilage cells, consistent with previous reports. . $^{37,41,45}$

\section{Processing of Samples for Histological and Immunohistochemical Analysis}

All procedures performed in mice were conducted in accordance with the policies and guidelines proposed in an animal protocol approved by Institutional Animal Care and Use Committee. Adult mouse knee joints and lumbar spine tissues were prepared for histology as described previously. ${ }^{43,45}$ Briefly, adult mouse knee joints and lumbar spine tissues were fixed, decalcified, and embedded in either paraffin or optimal cutting temperature compound (OCT). Five- and $10-\mu \mathrm{m}$-thick sections were cut for paraffin and OCT blocks, respectively, and 4-6 serial sagittal paraffin sections were harvested every $30 \mu \mathrm{m}$ apart within the midsagittal region of knee joints and intervertebral bodies. Each group contained at least 6 mice; 3 sections from each sample were stained with Safranin-O-fast green (proteoglycan is red) and Alcian blue- $\mathrm{H} \& \mathrm{E}$ (cartilage is blue) as described previously. ${ }^{44,45}$ Phenotype was evaluated based on classification of age-related changes in lumbar intervertebral discs. ${ }^{7}$ OCT sections were stained with antiCTGF (ab6992, abcam) as described previously. ${ }^{45}$ 

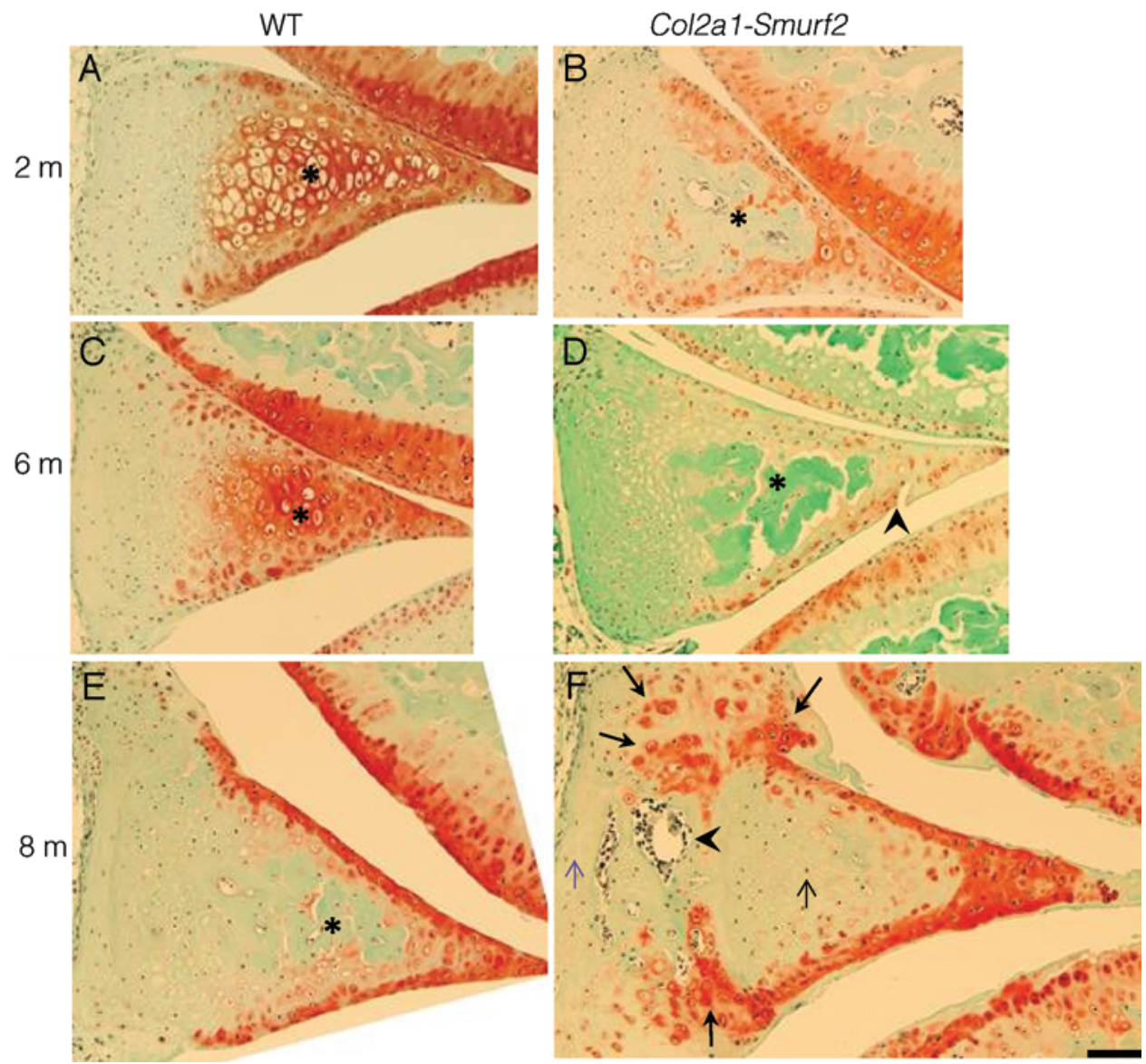

FIG. 1. Meniscus aging in wild-type (WT) mice and degeneration in Col2a1-Smurf2 transgenic mice. Safranin-O staining was performed on sagittal sections from the medial joint compartment of WT (A, C, and E) and Col2a1-Smurf2 transgenic mice (B, D, and $\mathrm{F}$ ) at various ages as indicated. A-D: The meniscus inner zone underwent calcification in Col2a1-Smurf2 transgenic mice at 2 and 6 months of age. Asterisks in panels $A$ and $C$ represented articular cartilage-like tissue in the inner zone of WT meniscus; asterisks in panels $B$ and $D$ represent calcified cartilage matrix in the inner zone of transgenic meniscus. $E$ and F: Meniscus aging in WT and degeneration progression in Col2a1-Smurf2 transgenic mice at 8 months of age. Calcification occurred in the inner one-third of WT meniscus ( $\mathrm{E}$, asterisk) (6 of 9 specimens). Fibrotic tissue occurred in the inner one-third of transgenic meniscus ( $\mathrm{F}$, thin black arrow), similar to the fibrotic tissue in the meniscus root ( $\mathrm{F}$, thin blue arrow). Thick black arrows and arrowhead in panel $\mathrm{F}$ indicate the chondrogenesis and ossification center, respectively. Bar $=100 \mu \mathrm{m}$. Figure is available in color online only.

\section{Western Blot Analysis}

Radioimmunoprecipitation assay (RIPA) buffer was used to extract proteins from disc cells that were freshly isolated without culture. We used a modified method for isolation of disc cells based on previous reports. ${ }^{3,13}$ Briefly, lumbar discs were isolated from 6 mice in each group under a dissecting microscope and digested with $0.2 \%(\mathrm{w} / \mathrm{v})$ Pronase (Roche) at $37^{\circ} \mathrm{C}$ for 1 hour and then with $0.02 \%$ (w/v) collagenase (Sigma) in DMEM/F-12. Cells were harvested by filtering the digested mixture through a $70-\mu \mathrm{m}$ strainer. Proteins of 10-15 $\mu \mathrm{g}$ were separated by SDSPAGE, blotted with anti-CTGF antibody (ab6992, abcam), and visualized through exposure of BioMax XAR film (Carestream).

\section{Real-Time Reverse Transcription Polymerase Chain Reaction}

Total RNA was extracted by using TRIzol from cells similar to those used for Western blot. RNA was reverse transcribed into cDNA using SuperScript IV Reverse
Transcriptase (ThermoFisher Scientific). Real-time reverse transcription polymerase chain reaction (RTPCR) was performed as previously described ${ }^{45}$ by using SYBR Green. The primers for CTGF were $5^{\prime}$-CTG TCAAGTTTGAGCTTTCTGG-3' and 5'-GGACTCAAA GATGTCATTTGTCC- $3^{\prime} ; 2$ the primers for TGF- $\beta 1$ were 5'-TGCTCGCTTTGTACAACAGCA-3' and 5'-GGTTTT CTCATAGATGGCGTTG-3'.

\section{Results}

\section{Meniscus Aging and Degeneration in Wild-Type and Col2a1-Smurf2 Transgenic Mice}

We previously showed that ectopically expressed Smurf2 driven by the Col2al promoter induces articular cartilage degeneration and osteophyte formation in adult knee joints in Col2al-Smurf2 transgenic mice. ${ }^{43,45}$ Meanwhile, we also found that meniscus degeneration progresses at a pace similar to that of articular cartilage degeneration in these mice (Fig. 1). ${ }^{45}$ Specifically, in the wild-type 
(WT) mice at 2 and 6 months of age, the articular cartilage-like tissue in the inner one-third of the meniscus contained rounded chondrocytes, which were embedded in the cartilage matrix (Fig. 1A and C). However, the proteoglycan content was decreased and the cartilage matrix was calcified in the corresponding region of 2-month-old Col2al-Smurf2 transgenic mice (Fig. 1B), and these phenotypic changes had progressed in 6-month-old transgenic mice. For example, the area with a decrease of proteoglycan content and calcification of cartilage matrix was expanded, and clefts had occurred in the meniscus cartilage surface (Fig. 1D). In 8-month-old WT mice, proteoglycan decrease and matrix calcification occurred in the inner articular cartilage-like tissue of menisci (Fig. 1E), the degree of which was similar to that in 2-month-old transgenic mice (Fig. 1, compare $\mathrm{E}$ with B). In the age-matched Col2al-Smurf2 transgenic mice, however, the inner onethird region, which was normally cartilage-like, turned out to be fibrotic tissue, similar to that in the meniscus root (Fig. 1F). Chondrogenesis and endochondral ossification (Fig. 1F) were often seen in the outer fibrocartilage area and resulted in an expanded outer region of meniscus and osteophytes around the joints. ${ }^{45}$ These data suggest that 1) meniscus degeneration is an age-related process in WT mice; and 2) ectopic expression of Smurf2 in Col2alpromoter working cells accelerates age-related meniscus degeneration in Col2al-Smurf2 transgenic mice.

\section{Intervertebral Disc Aging and Degeneration in WT and Col2a1-Smurf2 Transgenic Mice}

Given that ectopic expression of Smurf2 in Col2alpromoter working cells induced meniscus degeneration in Col2al-Smurf2 transgenic mice, the similarities between the meniscus and intervertebral disc prompted us to examine the phenotypic changes of intervertebral discs in these transgenic mice. We harvested lumbar spines from mice at various ages and performed histological and immunohistochemical analysis of the specimens. In 2-month-old WT mice, the matrix in the outer annulus was densely organized into lamellae, and the cells were fusiform in shape (Fig. 2A). ${ }^{9}$ The matrix in the inner annulus was loosely packed, and the cells were spherical. ${ }^{20}$ The matrix in the nucleus was a gel-like tissue, with loosely packed Type II collagen fibrils. The endplate was a layer of articular cartilage-like tissue, where the superficial zone contained Type II collagen, proteoglycans, and small flat cells (Fig. 2A; also see Fig. 4C). Compared with the discs of 2-month-old WT mice, discs of the age-matched Col2al-Smurf2 transgenic mice exhibited decreased proteoglycan content in the inner annulus, nucleus, and superficial zone of the endplate and increased endplate thickness (Fig. 2B). In 6-monthold WT mice, the intervertebral disc structure and matrix components were similar to those in the 2-month-old WT mice (Fig. 2C). In the age-matched Col2al-Smurf2 transgenic mice, however, tears in the annulus and matrix breakdown in the nucleus and endplate were often detected (Fig. 2D). Proteoglycan content in the inner annulus and nucleus was dramatically increased compared with that in the WT counterparts (Fig. 2, compare D with C), which was usually considered to be an attempt to initiate repair, ${ }^{2}$ similar to the alterations in articular cartilage from these transgenic mice. ${ }^{45}$ In the WT mice that were 10 months of age or older, more than one-third exhibited some disc degeneration (aging). For example, an increase in proteoglycan content in the inner annulus and nucleus was detected in 10-month-old mice ( $n=3$ of 8 ) (Fig. 2E), and annulus tears and nucleus matrix breakdown occurred in 12-month-old mice ( $n=3$ of 6 ) (Fig. 2G). These phenotypic changes in aging discs of the 10- to 12-month-old WT mice were similar to those in 6-month-old Col2al-Smurf2 transgenic mice (Fig. 2, compare E and G with D). In contrast to the focal and minor disc degeneration (disc aging) seen in the 10- to 12-month-old WT mice, 10-month-old Col2al-Smurf2 transgenic mice exhibited entire nucleus degradation (Fig. 2F). By 12 months, the nucleus degradation had expanded beyond the endplate into the calcified region of the vertebra (Fig. $2 \mathrm{H}$ ). These data indicate that intervertebral disc degeneration is an age-related process, ${ }^{6}$ and ectopic expression of Smurf2 in Col2al-promoter working cells greatly accelerates the degenerative process in Col2al-Smurf2 transgenic mice.

\section{Abnormal Chondrocyte Differentiation and Maturation Leads to Progression of Disc Degeneration in Col2a1-Smurf2 Transgenic Mice}

To better understand the process of disc degeneration from nucleus breakdown at 10 months of age to expansion of it beyond the endplate into vertebra at 12 months of age in Col2al-Smurf2 transgenic mice, we histologically examined serial disc sections from 11-month-old mice. Chondrocytes in the endplates underwent proliferation and formed column-like structures that migrated into the nucleus (Fig. 3B and $\mathrm{B}^{\prime}$ ). Clefts and tears occurred in the front area of the migrating tissue, and scar/fibrotic tissue started to develop below the clefts and tears (Fig. $\left.3 \mathrm{~B}^{\prime}\right)$. Similarly, chondrocyte-like cells in the inner annulus also underwent proliferation, forming cell clusters (Fig. 3C' and $\mathrm{D}^{\prime}$ ), which transformed into large hypertrophic chondrocyte-like cells and migrated into the nucleus. Wide tears or tissue breakdown occurred within the "hypertrophic-like area" containing many hypertrophic cells, and fibrotic tissue was formed adjacently (Fig. $3 \mathrm{C}^{\prime}$ and $\mathrm{D}^{\prime}$ ). In parallel with the cartilaginous formation in the inner annulus and endplates, cells in the outer annulus, which are normally fibroblast-like and predominantly express Type I collagen, differentiated into chondrocyte-like cells with expression of cartilage matrix proteins such as proteoglycans and Type II collagen (Figs. 2H, 3C, and 4F). In 8-month-old Col2al-Smurf2 transgenic mice, when disc degeneration was at an early stage (i.e., loss of the annulus nucleus boundary [Fig. 4B] and minor breakdown of the superficial layer of the endplate [Fig. 4D]), we observed that the outer annulus cells became big and rounded, but without strong expression of Type II collagen (Fig. 4, compare B with A). These data, along with data from the 12-month-old Col2al-Smurf2 transgenic mice, indicate that the degree of fibroblast differentiation into chondrocytes is parallel with the degenerative grade of discs.

\section{CTGF Expression Is Increased in Intervertebral Discs in Col2a1-Smurf2 Transgenic Mice}

Given the role of CTGF in chondrocyte maturation, ${ }^{22,26,28}$ 

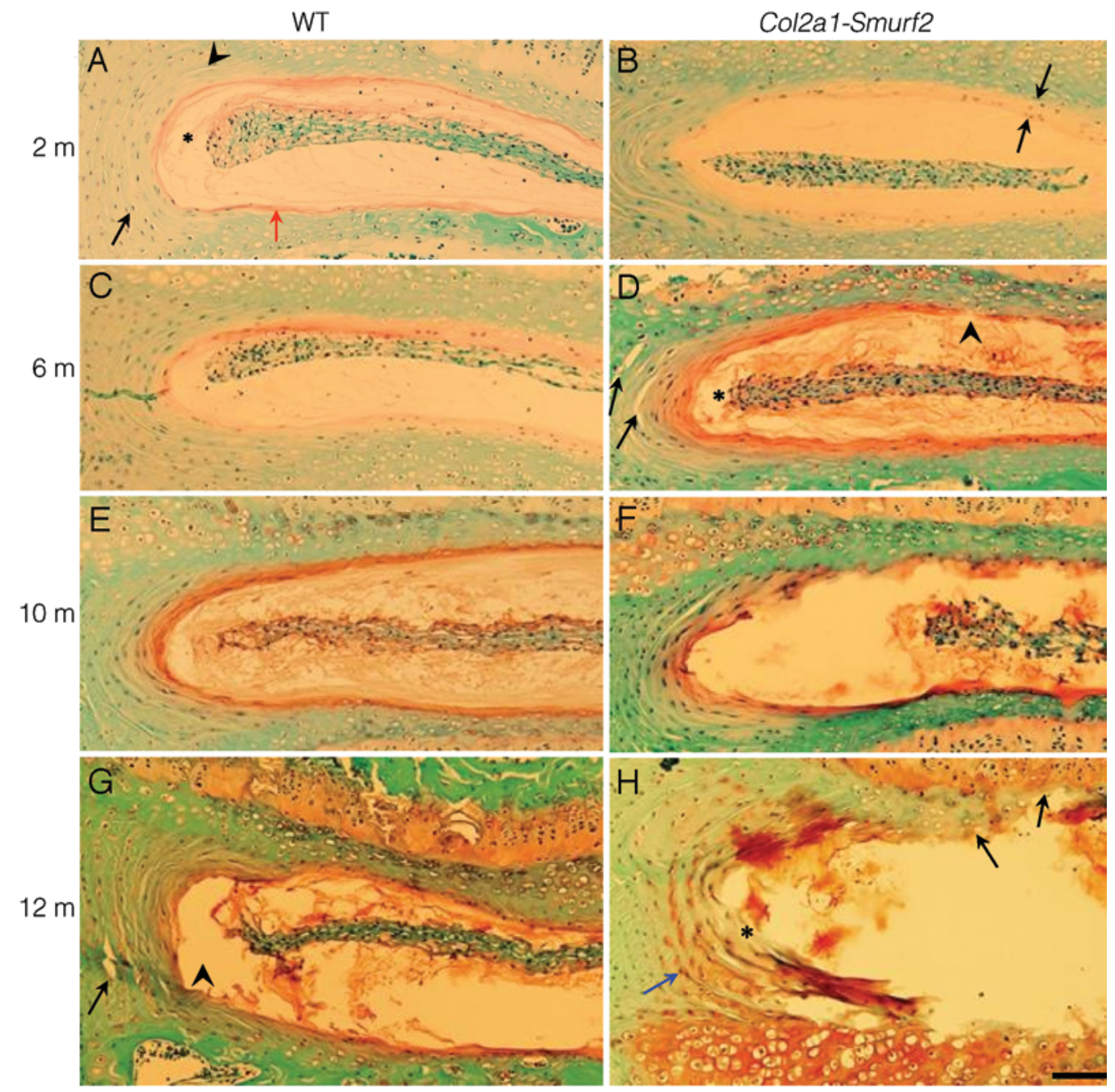

FIG. 2. Lumbar disc degeneration in Col2a1-Smurf2 transgenic mice. Safranin-O staining of lumbar intervertebral discs from WT $(A, C, E$, and $G$ ) and Col2a1-Smurf2 transgenic mice $(B, D, F$, and $H)$ at various ages as indicated. $A$ and $B$ : Proteoglycan content was decreased in transgenic discs in 2-month-old mice. In WT mice $(A)(n=7)$, the matrix in the outer annulus was densely organized lamellae with spindle-like cells and that in the inner annulus was loosely packed (A, arrowhead) with spherical cells ( $A$, black arrow). The superficial zone of the endplate was a thin layer of proteoglycan-rich tissue (A, red arrow). The nucleus was a gel-like tissue predominantly containing proteoglycans (stained to be red) with loosely packed Type II collagen fibrils (A, asterisk). In the transgenic mice $(B)(n=7)$, the thickness of endplate was increased (B, black arrows); the proteoglycan content in the inner annulus, nucleus, and endplates was decreased. $\mathrm{C}$ and $\mathbf{D}$ : Compensatory proteoglycan synthesis in transgenic mice at 6 months of age. Compared with WT disc (C), the proteoglycan content in transgenic disc (D) was increased in the inner annulus, nucleus, and endplates $(n=7)$. Arrows in $D$ indicate annulus tears; arrowhead and asterisk indicate matrix breakdown in the endplate and nucleus, respectively. E and F: Mild disc aging in WT and nucleus degradation in transgenic mice at 10 months of age. Three of 8 WT mice exhibited increased proteoglycan content in the inner annulus and nucleus (E). Transgenic mice exhibited severe nucleus degeneration $(F)(n=6)$. G and $H$ : Disc aging in WT and severe disc degeneration in transgenic mice at 12 months of age. In WT mice $(G), 3$ of 6 mice exhibited annulus tears ( $G$, arrow) and nucleus matrix breakdown ( $G$, arrowhead). In transgenic mice $(H, n=$ $6)$, nucleus degradation expanded beyond the endplate to the growth plate of vertebra $(\mathrm{H}$, arrows). Asterisk in $(\mathrm{H})$ indicates inner annulus undergoing degeneration. Blue arrow in $(\mathrm{H})$ indicates fibroblast-chondrocyte differentiation in the outer annulus. Bar $=100$ $\mu \mathrm{m}$. Figure is available in color online only.

wound healing, and fibrotic disease, ${ }^{19,17}$ formation of scar tissue and fibrosis in the vicinity of structural defects observed in lumbar discs of Col2al-Smurf2 transgenic mice could be due to an increase in CTGF expression levels. To test this hypothesis, we examined CTGF expression levels in lumbar discs by using immunohistochemical, Western blot, and real-time RT-PCR analyses. Immunostaining of specimens from 6-month-old WT mice with anti-CTGF antibody revealed that fibroblast-like cells in the outer an- nulus expressed a moderate level of CTGF (Fig. 5A), and this staining was barely detectable in the inner annulus and boundary cells. In the corresponding regions from the age-matched Col2al-Smurf2 transgenic mice, however, much higher CTGF expression levels were detected in the chondrocyte-like cells differentiated from fibroblast-like cells in the outer annulus and in the enlarged inner annulus cells, which are normally small and spherical (Fig. 5B). By 10 months, these changes were more pronounced 

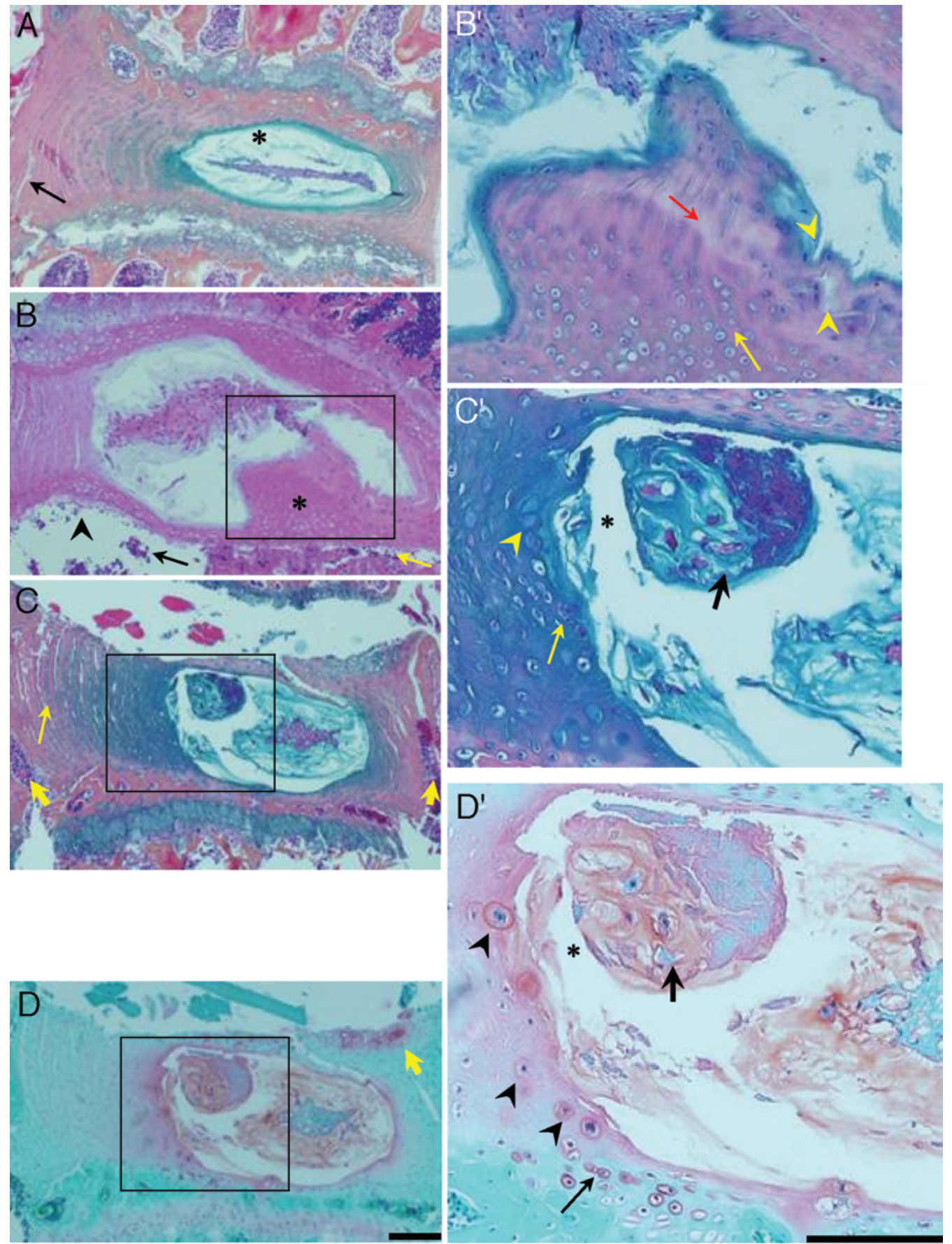

FIG. 3. Abnormal chondrocyte proliferation and maturation leads to disc degeneration progression in Col2a1-Smurf2 transgenic mice. A-C: Sections were stained with Alcian blue-H \& E (AHE). In 11-month-old WT mice (A), AHE staining detected outer annulus tears (A, arrow) and nucleus matrix degradation (A, asterisk) in intact discs. In 11-month-old transgenic mice (B and $C)$ ( $B$ ' and $C^{\prime}$ are higher-magnification images of boxed areas in $B$ and $C$ ), endplate chondrocytes underwent proliferation and formed column-like structures and migrated into the nucleus (B, asterisk; $B^{\prime}$, yellow arrow); clefts and tears were formed in the front area of the cartilaginous tissue ( $\mathrm{B}^{\prime}$, yellow arrowheads); and fibrotic tissue started to form below the clefts and tears ( $\mathrm{B}^{\prime}$, red arrow). Annulus chondrocyte-like cells formed cell clusters ( $C^{\prime}$, yellow arrow), within which the cells retained their hypertrophic characteristics ( $C^{\prime}$, arrowhead). Wide tears occurred within the hypertrophic-like tissue $\left(\mathrm{C}^{\prime}\right.$, asterisk), and fibrotic tissue was formed adjacently $\left(C^{\prime}\right.$, thick arrow). Thin arrow and thick arrows in $\mathrm{C}$ indicate fibroblast-chondrocyte differentiation in the outer annulus and bony tissue in the outmost annulus, respectively. D: Consecutive section of $C$ but stained with Safranin-O fast green. Thick arrow in D represents maturing cartilage in the connective area between the vertebra epiphysis and outer annulus. Thin arrow, arrowheads, asterisk, and thick arrow in D' represent structures similar to those in $C^{\prime}$. Bars $=100 \mu \mathrm{m}$. Figure is available in color online only. 


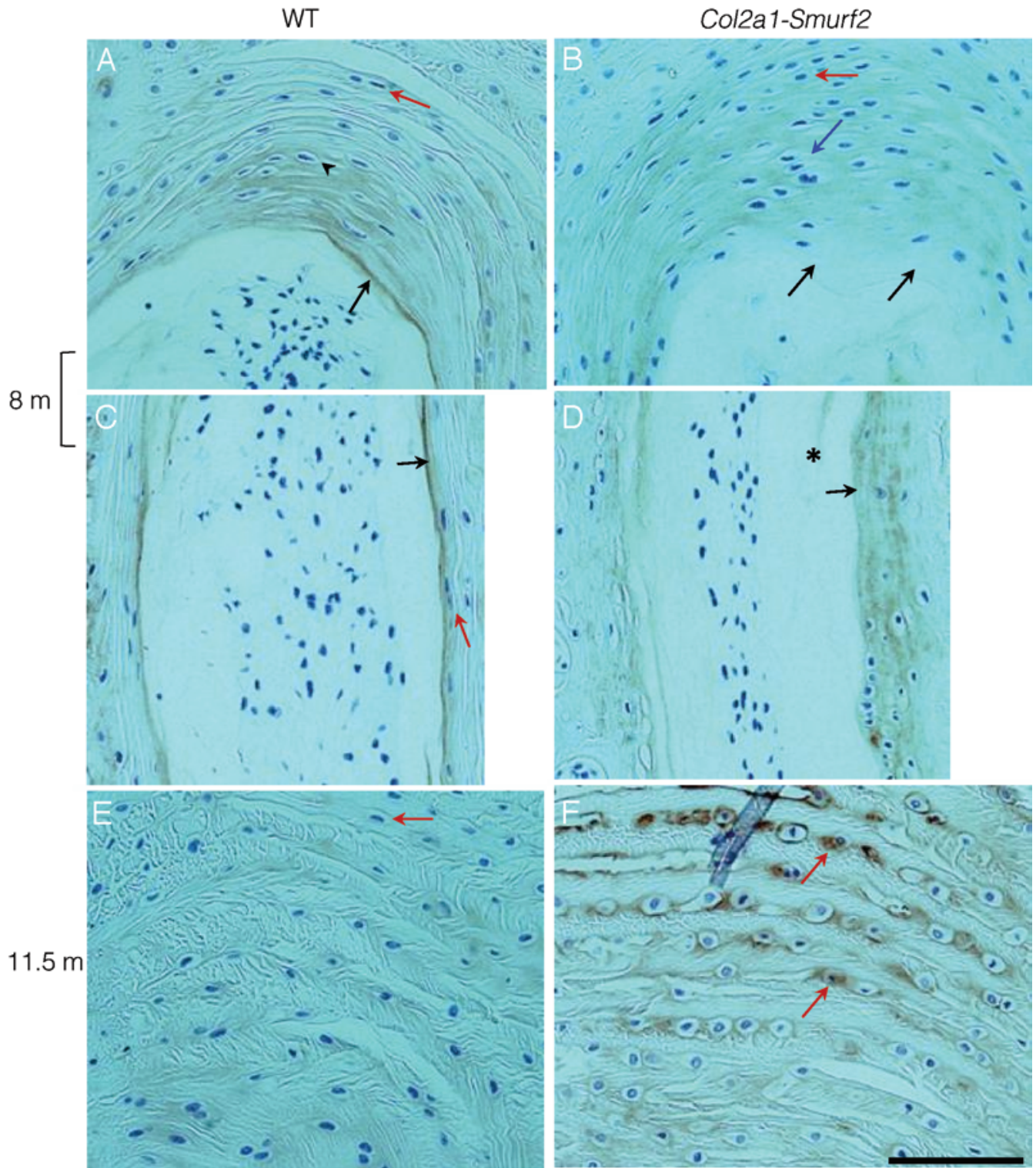

FIG. 4. Altered expression of Type II collagen in Col2a1-Smurf2 transgenic intervertebral discs. A and C: Type II collagen expression pattern in 8-month-old WT mouse discs. Images in panels A and C are from one section. Type II collagen was high in the annulus-nucleus boundary ( $\mathrm{A}$, black arrow) and superficial layer of the endplate ( $C$, black arrow). Spherical cells in the inner annulus expressed and deposited moderate levels of Type II collagen (A, arrowhead). Type II collagen was barely detected in spindle-like cells in the outer annulus (A, red arrow). Cells located under the superficial layer of the endplate were small and flat (C, red arrow). B and D: Loss of an annulus-nucleus boundary and superficial layer of endplate in 8-month-old transgenic mouse discs. Images in panels B and D are from one section. The annulus-nucleus boundary and superficial layer with rich Type II collagen were lost (B and D, black arrows). Spherical cells in the inner annulus became big and rounded, and Type II collagen production and deposition were reduced ( $B$, blue arrow). The outer annulus cells became rounded chondrocyte-like cells, but without obvious Type II collagen expression (B, red arrow). Asterisk in D indicates the nucleus. E and F: Fibroblasts differentiated into chondrocyte-like cells in 12-month-old transgenic mouse discs. The spindle-like cells in the outer annulus barely expressed Type II collagen in WT discs (E, red arrow). The cells in the corresponding region of transgenic discs became rounded chondrocyte-like cells with expression of Type II collagen (F, red arrows). Bar $=100 \mu \mathrm{m}$. Figure is available in color online only.

in the inner annulus, i.e., more rounded chondrocyte-like cells with stronger CTGF staining in this area (Fig. 5D).

To confirm the immunohistochemical result, we analyzed CTGF protein and mRNA levels in freshly isolated disc cells from transgenic and WT mice (Fig. 5E). Western blot showed that the protein levels of CTGF $(36-38 \mathrm{kD})^{8}$ in disc cells from 6- and 10-month-old Col2al-Smurf2 transgenic mice were significantly higher than those from the corresponding WT controls (Fig. 5E). Consistently, the mRNA levels of CTGF in the similar transgenic disc cells were 3 -fold higher than those in the corresponding WT controls (Fig. 5G). Furthermore, the levels of CTGF protein and mRNA in 10-month-old WT mice were slightly higher than those in 6-month-old WT mice, and a similar 


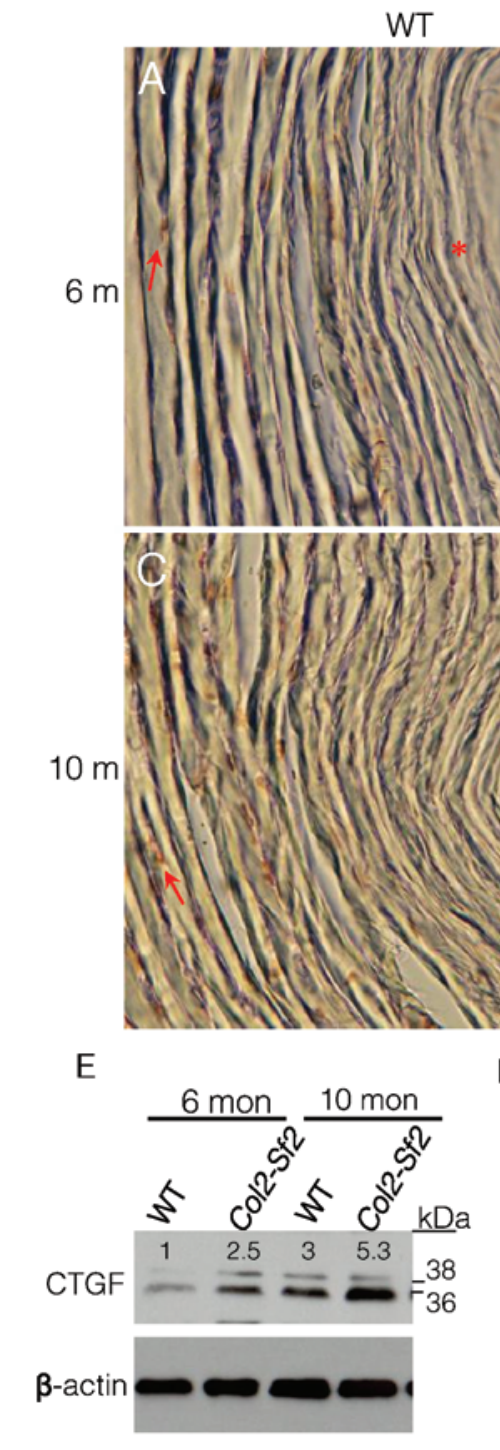

\section{Col2a1-Smurf2}
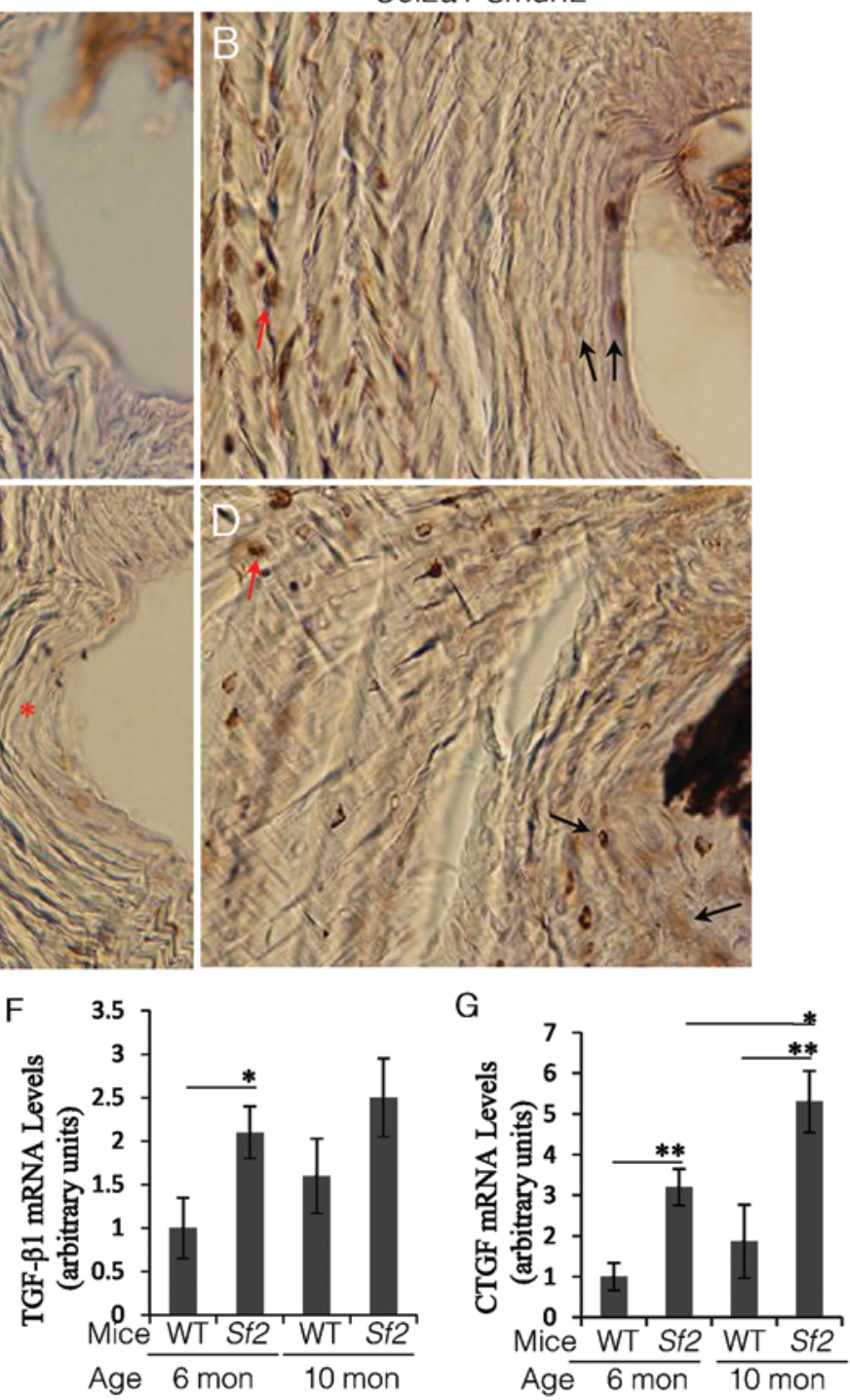

FIG. 5. Increase in CTGF expression in Col2a1-Smurf2 transgenic lumbar discs. A-D: Immunostaining of frozen sections with anti-CTGF antibodies showed that CTGF expression levels were increased in discs of 6- to 10-month-old transgenic mice. In 6- and 10-month-old WT mice (A and C), low or moderate levels of CTGF expression were detected in the outer fibroblast-like cells (red arrow); CTGF was rarely detected in the inner and boundary cells (asterisk). In age-matched transgenic mice (B and D), however, CTGF expression levels were strongly detected in the chondrocyte-like cells that were differentiated from fibroblasts in the outer annulus (red arrow) and were frequently detected in the inner and boundary cells (black arrows). Original magnification $\times 40$. E-G: CTGF protein and mRNA levels were increased in Col2a1-Smurf2 transgenic disc cells. Protein extracts or total RNA were prepared from disc cells isolated from lumbar discs of 6 WT and 6 transgenic mice without going through cell culture. E: Increase in CTGF protein levels in transgenic disc cells was shown by Western blot. Ten- to $15-\mu \mathrm{g}$ proteins were separated by SDS-PAGE and blotted with anti-CTGF antibodies. The values above the blotting bands show results of densitometric analysis of the Western blot (normalized to $\beta$-actin). F and G: Slight increase in TGF $\beta 1$ (F) and significant increase in CTGF (G) mRNA levels were shown by real-time RT-PCR. Values are the mean and SEM of 3 independent experiments. Statistical analysis was conducted using 1-way ANOVA and Student unpaired t-test. ${ }^{*} p<0.05 ;{ }^{* *} p<0.01$. Figure is available in color online only.

pattern was detected in 10- and 6-month-old transgenic mice (Fig. 5E and G), suggestive of a trend toward increased CTGF expression levels with increasing age and degenerative grade, consistent with previous results in human and mouse discs. . $, 33,38^{-10}$

Given the ability of TGF $\beta$ to induce CTGF expression in many types of cells, ${ }^{19,21,38}$ we examined whether TGF $\beta$ mRNA levels were increased in the Col2al-Smurf2 transgenic disc cells versus WT control cells. Indeed, a small but significant increase in the TGF $\beta 1 \mathrm{mRNA}$ level was detected in transgenic disc cells from 6- but not 8-month-old mice versus WT control cells (Fig. 5F).

\section{Discussion}

During the process of osteoarthritis development in Col2al-Smurf2 transgenic mice, ${ }^{44,45}$ the cartilage-like tissue in the inner meniscus demonstrated similar alternations 
in young and adult mice, including matrix degradation and calcification, and the outer meniscus root, which is fibrotic tissue near the synovial membrane and capsule, exhibited chondrogenesis and ossification in old mice (Fig. 1F). It appeared that the meniscus degeneration in the transgenic mice was a primary, not a secondary, consequence of articular cartilage degeneration, because the degeneration in the articular cartilage and menisci occurred and progressed almost simultaneously.

Given the similarities between menisci and intervertebral discs, the degenerative phenotype in menisci in Col2al-Smurf2 transgenic mice prompted us to examine the disc phenotype in transgenic mice. By comparing cell phenotype and matrix composition and architecture in Col2al-Smurf2 transgenic mouse discs with those in WT controls at various ages (Figs. 2-4), we found that intervertebral discs underwent physiological aging in WT mice, and ectopic expression of Smurf 2 driven by the Col2al promoter accelerated this aging process by 3-6 months, leading to disc degeneration. Although rodent models of disc degeneration have an increased propensity for disc repair due to high cell density and the presence of notochordal cells in the adult nucleus, Col2a1-Smurf 2 transgenic mice exhibited a chronic disc degeneration phenotype. During the development of disc degeneration in this mouse model, we observed that many microscopic changes, such as fibroblast-to-chondrocyte differentiation, cell cloning, migration, and fibrosis, were similar to those occurring in humans. ,, $33^{2}$ These similarities are probably attributable to the following: 1) The level of ectopic Smurf2 expression driven by the Col2al promoter in Col2a1-Smurf2 transgenic mouse chondrocytes was only 2.6-fold greater than that in WT controls, ${ }^{45}$ which might be just over a threshold level of Smurf2 expression in disc cells that can initiate certain molecular events associated with disc aging or degeneration progression. 2) Cells with ectopic Smurf2 expression driven by Col2al promoter were chondrocyte-like cells in the inner annulus for young adult Col2al-Smurf 2 transgenic mice and differentiated chondrocyte-like cells in the outer annulus for old transgenic mice. Therefore, most of the annulus cells in old Col2al-Smurf2 transgenic mice were chondrocytelike and expressed the transgene Smurf2, characteristics similar to those observed in cells in human degenerative discs, in which both inner annulus and nucleus cells were chondrocyte-like. .,10,33 $^{-3}$

Given the role of CTGF in wound healing and fibrotic disease through its promotion of cell adhesion, migration, and proliferation along with matrix production and fibrosis formation, ${ }^{10,17,19}$ we speculated that the phenotypic changes such as chondrocyte-like cell clusters, migration of cartilaginous tissue into nucleus, and formation of fibrosis-like tissues in degenerative discs (Fig. 3) could be a result of increased CTGF levels in the onsite cells or the cells that were prone to becoming degenerative or fibrotic tissue. As expected, stronger staining for CTGF and higher CTGF protein and mRNA levels were detected in Col2al-Smurf2 transgenic discs than in WT control discs (Fig. 5). Consistent with the CTGF expression pattern, TGF $\beta$ mRNA levels detected in the same transgenic discs were higher than those in WT control discs (Fig. 5F), although the increase was small or not significant. This finding is probably attributable to the following circumstances: TGF $\beta$ expression level does not always correlate with the levels of its biologically active polypeptides in the matrix environment due to posttranscriptional mechanisms ${ }^{25,32}$ and CTGF can increase TGF $\beta$ activity by enhancing TGF $\beta$ binding to its receptors. ${ }^{1}$ Overall, when structural defects occur at an early stage of disc degeneration, disc cells attempt to initiate repair by activating chondrocyte maturation and matrix production, as well as $C T G F$ gene expression. However, because the regenerated cartilaginous tissue cannot resist mechanical loading from daily activities, the result may be expansion of structural failure and persistence of $C T G F$ expression, eventually leading to disc scarring and fibrosis.

\section{Conclusions}

Ectopic expression of Smurf2 under the control of Col2al promoter in mice induced disc degeneration by accelerating the age-related disc aging process. In addition, many phenotypic changes such as fibroblast-chondrocyte differentiation, chondrocyte cloning, and fibrotic tissue formation observed in Col2al-Smurf 2 transgenic degenerative discs were frequently detected in humans, and CTGF protein and mRNA levels were upregulated in these chondrocyte-like cells in Col2al-Smurf 2 transgenic discs. Thus, our findings indicate that Smurf2-mediated disc degeneration occurs via upregulation of CTGF, and this pathway may represent a novel mechanism for initiation or progression of disc degeneration in human beings.

\section{Acknowledgments}

This study was supported by grants to Dr. Jason H. Huang from NIH/NINDS (R01 NS067435), Baylor Scott \& White Plummer, and Baylor Scott \& White Equipment. Col2al promoter was provided by Yoshihiko Yamada (NIH/NIDCR); FLAG-tagged human Smurf 2 cDNA was purchased from Addgene. The research data in this study were extensively reviewed and supported for publication by Randy Rosier (University of Rochester). The manuscript was extensively reviewed by Quoc-Bao Nguyen (Texas A\&M College of Medicine).

\section{References}

1. Abreu JG, Ketpura NI, Reversade B, De Robertis EM: Connective-tissue growth factor (CTGF) modulates cell signalling by BMP and TGF- $\beta$. Nat Cell Biol 4:599-604, 2002

2. Adams MA, Roughley PJ: What is intervertebral disc degeneration, and what causes it? Spine (Phila Pa 1976) 31:21512161,2006

3. Aguiar DJ, Johnson SL, Oegema TR: Notochordal cells interact with nucleus pulposus cells: regulation of proteoglycan synthesis. Exp Cell Res 246:129-137, 1999

4. Antoniou J, Steffen T, Nelson F, Winterbottom N, Hollander AP, Poole RA, et al: The human lumbar intervertebral disc: evidence for changes in the biosynthesis and denaturation of the extracellular matrix with growth, maturation, ageing, and degeneration. J Clin Invest 98:996-1003, 1996

5. Bandyopadhyay A, Tsuji K, Cox K, Harfe BD, Rosen V, Tabin CJ: Genetic analysis of the roles of BMP2, BMP4, and BMP7 in limb patterning and skeletogenesis. PLoS Genet 2:e216, 2006

6. Bedore J, Sha W, McCann MR, Liu S, Leask A, Séguin CA: Impaired intervertebral disc development and premature 
disc degeneration in mice with notochord-specific deletion of CCN2. Arthritis Rheum 65:2634-2644, 2013

7. Boos N, Weissbach S, Rohrbach H, Weiler C, Spratt KF, Nerlich AG: Classification of age-related changes in lumbar intervertebral discs: 2002 Volvo Award in basic science. Spine (Phila Pa 1976) 27:2631-2644, 2002

8. Bradham DM, Igarashi A, Potter RL, Grotendorst GR: Connective tissue growth factor: a cysteine-rich mitogen secreted by human vascular endothelial cells is related to the SRCinduced immediate early gene product CEF-10. J Cell Biol 114:1285-1294, 1991

9. Bruehlmann SB, Rattner JB, Matyas JR, Duncan NA: Regional variations in the cellular matrix of the annulus fibrosus of the intervertebral disc. J Anat 201:159-171, 2002

10. Chan WC, Sze KL, Samartzis D, Leung VY, Chan D: Structure and biology of the intervertebral disk in health and disease. Orthop Clin North Am 42:447-464, vii, 2011

11. Chen M, Zhu M, Awad H, Li TF, Sheu TJ, Boyce BF, et al: Inhibition of $\beta$-catenin signaling causes defects in postnatal cartilage development. J Cell Sci 121:1455-1465, 2008

12. Cheung KM, Karppinen J, Chan D, Ho DW, Song YQ, Sham P, et al: Prevalence and pattern of lumbar magnetic resonance imaging changes in a population study of one thousand forty-three individuals. Spine (Phila Pa 1976) 34:934-940, 2009

13. Collin EC, Grad S, Zeugolis DI, Vinatier CS, Clouet JR, Guicheux JJ, et al: An injectable vehicle for nucleus pulposus cell-based therapy. Biomaterials 32:2862-2870, 2011

14. Dagenais S, Caro J, Haldeman S: A systematic review of low back pain cost of illness studies in the United States and internationally. Spine J 8:8-20, 2008

15. Ghadially FN, Thomas I, Yong N, Lalonde JM: Ultrastructure of rabbit semilunar cartilages. J Anat 125:499-517, 1978

16. Girkontaite I, Frischholz S, Lammi P, Wagner K, Swoboda $\mathrm{B}$, Aigner T, et al: Immunolocalization of type $\mathrm{X}$ collagen in normal fetal and adult osteoarthritic cartilage with monoclonal antibodies. Matrix Biol 15:231-238, 1996

17. Gruschwitz M, Müller PU, Sepp N, Hofer E, Fontana A, Wick G: Transcription and expression of transforming growth factor type beta in the skin of progressive systemic sclerosis: a mediator of fibrosis? J Invest Dermatol 94:197203, 1990

18. Hellio Le Graverand MP, Ou Y, Schield-Yee T, Barclay L, Hart D, Natsume T, et al: The cells of the rabbit meniscus: their arrangement, interrelationship, morphological variations and cytoarchitecture. J Anat 198:525-535, 2001

19. Holmes A, Abraham DJ, Sa S, Shiwen X, Black CM, Leask A: CTGF and SMADs, maintenance of scleroderma phenotype is independent of SMAD signaling. J Biol Chem 276:10594-10601, 2001

20. Humzah MD, Soames RW: Human intervertebral disc: structure and function. Anat Rec 220:337-356, 1988

21. Igarashi A, Okochi H, Bradham DM, Grotendorst GR: Regulation of connective tissue growth factor gene expression in human skin fibroblasts and during wound repair. Mol Biol Cell 4:637-645, 1993

22. Ivkovic S, Yoon BS, Popoff SN, Safadi FF, Libuda DE, Stephenson RC, et al: Connective tissue growth factor coordinates chondrogenesis and angiogenesis during skeletal development. Development 130:2779-2791, 2003

23. McCann MR, Tamplin OJ, Rossant J, Séguin CA: Tracing notochord-derived cells using a Noto-cre mouse: implications for intervertebral disc development. Dis Model Mech 5:73-82, 2012

24. Messner K, Gao J: The menisci of the knee joint. Anatomical and functional characteristics, and a rationale for clinical treatment. J Anat 193:161-178, 1998

25. Millan FA, Denhez F, Kondaiah P, Akhurst RJ: Embryonic gene expression patterns of TGF beta 1, beta 2 and beta 3 suggest different developmental functions in vivo. Development 111:131-143, 1991

26. Nakanishi T, Nishida T, Shimo T, Kobayashi K, Kubo T, Tamatani T, et al: Effects of CTGF/Hcs24, a product of a hypertrophic chondrocyte-specific gene, on the proliferation and differentiation of chondrocytes in culture. Endocrinology 141:264-273, 2000

27. Nerlich AG, Schleicher ED, Boos N: 1997 Volvo Award winner in basic science studies. Immunohistologic markers for age-related changes of human lumbar intervertebral discs. Spine (Phila Pa 1976) 22:2781-2795, 1997

28. Nishida T, Kubota S, Nakanishi T, Kuboki T, Yosimichi G, Kondo S, et al: CTGF/Hcs24, a hypertrophic chondrocytespecific gene product, stimulates proliferation and differentiation, but not hypertrophy of cultured articular chondrocytes. J Cell Physiol 192:55-63, 2002

29. Oka M, Kubota S, Kondo S, Eguchi T, Kuroda C, Kawata K, et al: Gene expression and distribution of connective tissue growth factor (CCN2/CTGF) during secondary ossification center formation. J Histochem Cytochem 55:1245-1255, 2007

30. Pacifici M, Koyama E, Iwamoto M: Mechanisms of synovial joint and articular cartilage formation: recent advances, but many lingering mysteries. Birth Defects Res C Embryo Today 75:237-248, 2005

31. Pattappa G, Li Z, Peroglio M, Wismer N, Alini M, Grad S: Diversity of intervertebral disc cells: phenotype and function. J Anat 221:480-496, 2012

32. Pedrozo HA, Schwartz Z, Gomez R, Ornoy A, Xin-Sheng W, Dallas SL, et al: Growth plate chondrocytes store latent transforming growth factor (TGF)- $\beta 1$ in their matrix through latent TGF- $\beta 1$ binding protein-1. J Cell Physiol 177:343-354, 1998

33. Peng B, Chen J, Kuang Z, Li D, Pang X, Zhang X: Expression and role of connective tissue growth factor in painful disc fibrosis and degeneration. Spine (Phila Pa 1976) 34:E178-E182, 2009

34. Postacchini F, Bellocci M, Massobrio M: Morphologic changes in annulus fibrosus during aging. An ultrastructural study in rats. Spine (Phila Pa 1976) 9:596-603, 1984

35. Proctor CS, Schmidt MB, Whipple RR, Kelly MA, Mow VC: Material properties of the normal medial bovine meniscus. J Orthop Res 7:771-782, 1989

36. Roughley PJ, White RJ: The dermatan sulfate proteoglycans of the adult human meniscus. J Orthop Res 10:631-637, 1992

37. Shu B, Li TF, Li XF, Tang DZ, Zhang Y, Shi Q, et al: Chondrocyte-specific inhibition of $\beta$-catenin signaling leads to dysplasia of the caudal vertebrae in mice. Spine (Phila Pa 1976) 38:2079-2084, 2013

38. Tran CM, Markova D, Smith HE, Susarla B, Ponnappan RK, Anderson DG, et al: Regulation of CCN2/connective tissue growth factor expression in the nucleus pulposus of the intervertebral disc: role of Smad and activator protein 1 signaling. Arthritis Rheum 62:1983-1992, 2010

39. Verdonk PC, Forsyth RG, Wang J, Almqvist KF, Verdonk R, Veys EM, et al: Characterisation of human knee meniscus cell phenotype. Osteoarthritis Cartilage 13:548-560, 2005

40. von der Mark K, Kirsch T, Nerlich A, Kuss A, Weseloh G, Glückert K, et al: Type X collagen synthesis in human osteoarthritic cartilage. Indication of chondrocyte hypertrophy. Arthritis Rheum 35:806-811, 1992

41. Wang M, Tang D, Shu B, Wang B, Jin H, Hao S, et al: Conditional activation of $\beta$-catenin signaling in mice leads to severe defects in intervertebral disc tissue. Arthritis Rheum 64:2611-2623, 2012

42. Watanabe H, Yamada Y, Kimata K: Roles of aggrecan, a large chondroitin sulfate proteoglycan, in cartilage structure and function. J Biochem 124:687-693, 1998 
43. Wu Q, Chen D, Zuscik MJ, O'Keefe RJ, Rosier RN: Overexpression of Smurf2 stimulates endochondral ossification through upregulation of $\beta$-catenin. J Bone Miner Res 23:552-563, 2008

44. Wu Q, Huang JH, Sampson ER, Kim KO, Zuscik MJ, O'Keefe RJ, et al: Smurf2 induces degradation of GSK-3 $\beta$ and upregulates $\beta$-catenin in chondrocytes: a potential mechanism for Smurf2-induced degeneration of articular cartilage. Exp Cell Res 315:2386-2398, 2009

45. Wu Q, Kim KO, Sampson ER, Chen D, Awad H, O'Brien T, et al: Induction of an osteoarthritis-like phenotype and degradation of phosphorylated Smad3 by Smurf2 in transgenic mice. Arthritis Rheum 58:3132-3144, 2008

\section{Disclosures}

The authors report no conflict of interest concerning the materi- als or methods used in this study or the findings specified in this paper.

\section{Author Contributions}

Conception and design: both authors. Acquisition of data: Wu. Analysis and interpretation of data: both authors. Drafting the article: Wu. Critically revising the article: both authors. Reviewed submitted version of manuscript: both authors. Approved the final version of the manuscript on behalf of both authors: Wu. Statistical analysis: Wu. Administrative/technical/material support: Wu. Study supervision: $\mathrm{Wu}$.

\section{Correspondence}

Qiuqian Wu, Department of Neurosurgery, Institute for Translational Medicine, Baylor Scott \& White Health System, 5701 Airport Rd., MS-AR-D120, Temple, TX 76502. email: qiuqian.wu@bswhealth.org. 